\title{
Neural Mechanisms Underlying Melodic Perception and Memory for Pitch
}

\author{
Robert J. Zatorre, Alan C. Evans, and Ernst Meyer \\ McConnell Brain Imaging Centre, Montreal Neurological Institute, McGill University, Montreal, Quebec, Canada H3A 2B4
}

The neural correlates of music perception were studied by measuring cerebral blood flow (CBF) changes with positron emission tomography (PET). Twelve volunteers were scanned using the bolus water method under four separate conditions: (1) listening to a sequence of noise bursts, (2) listening to unfamiliar tonal melodies, (3) comparing the pitch of the first two notes of the same set of melodies, and (4) comparing the pitch of the first and last notes of the melodies. The latter two conditions were designed to investigate shortterm pitch retention under low or high memory load, respectively. Subtraction of the obtained PET images, superimposed on matched MRI scans, provides anatomical localization of CBF changes associated with specific cognitive functions. Listening to melodies, relative to acoustically matched noise sequences, resulted in CBF increases in the right superior temporal and right occipital cortices. Pitch judgments of the first two notes of each melody, relative to passive listening to the same stimuli, resulted in right frontal-lobe activation. Analysis of the high memory load condition relative to passive listening revealed the participation of a number of cortical and subcortical regions, notably in the right frontal and right temporal lobes, as well as in parietal and insular cortex. Both pitch judgment conditions also revealed CBF decreases within the left primary auditory cortex. We conclude that specialized neural systems in the right superior temporal cortex participate in perceptual analysis of melodies; pitch comparisons are effected via a neural network that includes right prefrontal cortex, but active retention of pitch involves the interaction of right temporal and frontal cortices.

[Key words: human auditory processing, music, pitch, auditory working memory, PET, temporal cortex, frontal cortex ]

Perceiving a melody, or sequence of pitches, requires relatively complex perceptual analysis, since short-term memory and abstract pattern-matching mechanisms must be involved (Deutsch, 1982; Dowling and Harwood, 1986). It seems likely that such processing would also demand a correspondingly complex set

\footnotetext{
Received July 8, 1993; accepted October 4, 1993.

We thank the technical staff of the McConnell Brain Imaging Unit and of the Medical Cyclotron Unit for their invaluable assistance, and P. Neelin, J. MorenoCantu, and S. Milot for their technical expertise. We also thank Dr. D. Perry for helpful discussions, and Dr. A. Gjedde for his guidance and support. Funding was provided in part by Grants MT 11541 and SP-30 from the Medical Research Council of Canada, by an award to R.J.Z. from the Fonds de la Recherche en Santé du Québec, and by the McDonneli-Pew Cognitive Neuroscience Center.

Correspondence should be addressed to Robert J. Zatorre, Montreal Neurologica! Institute, 3801 University Street, Montreal, Quebec, Canada H3A 2B4.

Copyright (C) 1994 Society for Neuroscience $0270-6474 / 94 / 141908-12 \$ 05.00 / 0$
}

of neural computations, but at present, the cerebral substrates for melodic processing remain poorly understood. Current knowledge of complex auditory neural processing comes from two principal sources: neuroanatomical and neurophysiological studies of the auditory system of nonhuman vertebrates, and studies of the effects of human brain lesions on auditory tasks. Each of these will be briefly reviewed before introducing the present investigation, which takes advantage of recent advances in PET brain imaging techniques to study the functional cerebral systems involved in melodic perception in the living human brain.

The superior aspect of the temporal lobe has long been known to contain neurons responsive to auditory stimulation in both monkey (Ferrier, 1876; Merzenich and Brugge, 1973) and human (Flechsig, 1896; Celesia, 1976). Several distinct cortical fields may be identified on the basis of the neurophysiological response properties of neurons within those fields (Brugge and Reale, 1985), and also based on their cytoarchitectonic characteristics. A general distinction may be drawn between the primary auditory area-or koniocortex-lying deep within Heschl's gyri in the human brain (Galaburda and Sanides, 1980; Liegeois-Chauvel et al., 1991), and surrounding regions, which may be termed secondary or associative auditory cortex. Some of these cortical ficlds are arranged tonotopically (Woolsey, 1971; see Brugge and Reale, 1985, for a review), suggesting that they play a role in frequency analysis. However, apart from the primary and immediately surrounding regions, the frequency tuning of individual units can be very broad, and the tonotopic organization can be difficult to discern (Manley and MüllerPreuss, 1978). Some neurons respond best to complex stimuli, such as tones modulated in frequency (Whitfield and Evans, 1965), or patterns of ascending or descending tone sequences (McKenna et al., 1989).

The morphological and physiological distinctions between cortical regions raise important questions regarding the consequences of such an architecture for auditory processing. Can dissociations be identified that would allow functional significance to be assigned to the structural heterogeneity? A possible functional role for human superior temporal neurons in higherorder auditory processes is suggested by the observations of Penfield and Perot (1963) and of Penfield and Jasper (1954). They noted that complex auditory sensations (including voices and music) could be elicited from elcctrical stimulation of the exposed cortex in this region, but not from Heschl's gyri, which instead usually resulted in more elementary sensations, such as buzzing.

The connectivity of these regions indicates that both primary and secondary areas receive input from various divisions of the medial geniculate nucleus (Burton and Jones, 1976), suggesting 
a probable parallel organization. This idea is supported by electrophysiological (Celesia, 1976) and behavioral (Tramo et al., 1990) observations indicating that secondary regions may continue to function even after destruction of primary areas. In addition, several investigators have described extensive projections from the superior temporal lobe to the anterior frontal cortex (Chavis and Pandya, 1976; Petrides and Pandya, 1988), which are topographically organized. Such connections imply that there may be a functional interaction between these cortices.

Another important issue that is particularly relevant to melodic processing concerns hemispheric specialization. The human neuropsychological literature remains somewhat controversial on this point. Although there is some consensus that many aspects of musical processing probably require contributions from neural systems within each cerebral hemisphere (Peretz, 1993), there is also considerable evidence indicating that right-hemisphere mechanisms are particularly important for some aspects of melody perception (for a more extensive review, see Marin and Perry, in press). Among the first to explore this issue systematically, Milner (1962) and Shankweiler (1966) demonstrated decrements in melodic discrimination following right temporal lobectomy, but not after left-sided removals. This finding was subsequently confirmed by Zatorre (1985) and by Samson and Zatorre (1988), who also found that left temporallobe removals could affect melodic discrimination, but only if the damage extended into portions of Heschl's gyri. Further consistent evidence was provided by Zatorre and Halpern (1993), who demonstrated deficits in pitch judgments of both perceived and imagined pitches within well-known songs after right, but not left, temporal-lobe excision.

Studies of single-tone pitch perception also generally support a preponderant role for right-hemisphere neural systems, but only in specific aspects of pitch processing. Thus, simple frequency discrimination is affected only slightly or not at all by unilateral cortical lesions (Milner, 1962; Zatorre, 1988), and can probably be accomplished via subcortical structures. The animal literature is also consistent with this assertion (Evarts, 1952; Jerison and Neff, 1953; Heffner and Masterton, 1978). However, if a pitch judgment requires spectral analysis, then right-side auditory cortical regions seem to play a special role. For example, perception of the missing fundamental is affected only by right temporal-lobe lesions that invade portions of Heschl's gyri, and not by more restricted anterior temporal-lobe damage or by left temporal excision (Zatorre, 1988). Similar findings have been reported in tasks requiring processing of complex harmonic structure (Sidtis and Volpe, 1988; Divenyi and Robinson, 1989; Robin et al., 1990). Furthermore, timbre discrimination tasks involving changes in harmonic structure have also yielded consistent evidence favoring right-asymmetric processing, both with temporal-lobe lesioned patients (Milner, 1962; Samson and Zatorre, 1991a), as well as with commissurotomized subjects (Tramo and Gazzaniga, 1989).

Short-term retention is another aspect of pitch processing that apparently requires asymmetric mechanisms. Bilateral ablations in the superior temporal gyrus of the monkey result in deficits in tonal retention (Stepien et al., 1960; Colombo et al., 1990). Single-unit data also implicate this region in auditory shortterm memory (Gottlieb et al., 1989). In human patients, Zatorre and Samson (1991) dcmonstrated that right temporal-lobe excision affected short-term memory for pitch when interfering stimuli were presented between the target and comparison items (Deutsch, 1970); they also observed that right frontal-lobe dam- age significantly impaired pitch retention, a finding mirrored in certain animal studies of bilateral frontal ablation (Gross and Weiskrantz, 1962; Iversen and Mishkin, 1973). The latter result may reflect some nonspecific effect of frontal lesions on behavior (e.g., disturbances in inhibition of responses), but a more intriguing possibility is that the disturbance is related to disruption of the frontotemporal connections discussed above, which would imply a role for the interaction between these cortices in pitch retention (Perry, 1990; Marin and Perry, in press; see also Chavis and Pandya, 1976).

The recent advent of functional PET activation techniques in normal human subjects has added one more source of information to the body of knowledge outlined above. To date, the majority of these studies have investigated verbal auditory processing, with the principal findings indicating increased cerebral blood flow (CBF) bilaterally in the superior temporal gyri while listening to verbal stimuli, and activation of specific left-hemisphere sites for certain aspects of phonological, lexical, or semantic processing (Petersen et al., 1988; Wise et al., 1991; Démonct ct al., 1992; Zatorre ct al., 1992). Activation of primary auditory regions for simple auditory stimuli such as pure tones (Lauter et al., 1985) and noise bursts (Zatorre et al., 1992) has also been reported. In one condition of our recent investigation (Zatorre et al., 1992) we investigated pitch processing by instructing subjects to compare the pitch of a pair of syllables; the pattern of CBF was subtracted from a prior condition in which the subjects listened to the same syllables but made no overt judgment. The principal result of this comparison was a CBF increase in two sites within the right inferior frontal cortex, which contrasted notably with CBF activation in left Broca's area when a phonetic judgment was required using the identical set of speech syllables. This result therefore implicates the right prefrontal cortex in pitch processing.

The present investigation was undertaken to explore melodic perceptual processes in normal human subjects via functional PET activation. Based on the studies described, we hypothesized that perceiving a novel tonal melody would entail neuronal processing in both left and right superior temporal regions, with a possibly greater contribution from the right side. We also predicted that secondary auditory cortices would be primarily involved, and therefore that activation of primary regions could be "subtracted out" by using a nonspecific auditory stimulus, matched for its acoustic characteristics with the melodies, as a control condition (Zatorre et al., 1992). Finally, we predicted that right frontal-lobe mechanisms would be engaged when subjects make specific judgments of pitch changes within a melody, and that when the judgment requires retention of pitch over a filled interval, additional right-temporal activity, beyond that initially present during passive listening, would be observed.

\section{Materials and Methods}

PET scanning. PET scans were obtained using the Scanditronix PC2048 system, which produces 15 brain image slices at an intrinsic resolution of $5.0 \times 5.0 \times 6.0 \mathrm{~mm}$ (Evans et al., 1991a). Using the bolus $\mathrm{H}_{2}{ }^{15} \mathrm{O}$ methodology (Raichle et al., 1983) without arterial blood sampling (Fox and Raichle, 1984), the relative distribution of CBF was measured in baseline and activated conditions. Individual high-resolution MRI studies (63 slices, $2 \mathrm{~mm}$ thick) were obtained from a Philips 1.5T Gyroscan and coregistered with the PET data (Evans et al., 1991b). An orthogonal coordinate frame was then established based on the anterior-posterior commissure line as identified in the MRI volume (Evans et al., 1992). These coordinates were used to apply a linear resampling of each matched pair of MRI and PET data sets into a 
Figure 1. A. Amplitude waveform of noise stimulus. $B$, Waveform of one melodic stimulus. Note similarity in duration and shape of noise bursts as compared to melodic stimulus. C; Examples of three melodies used as stimuli, in musical notation.
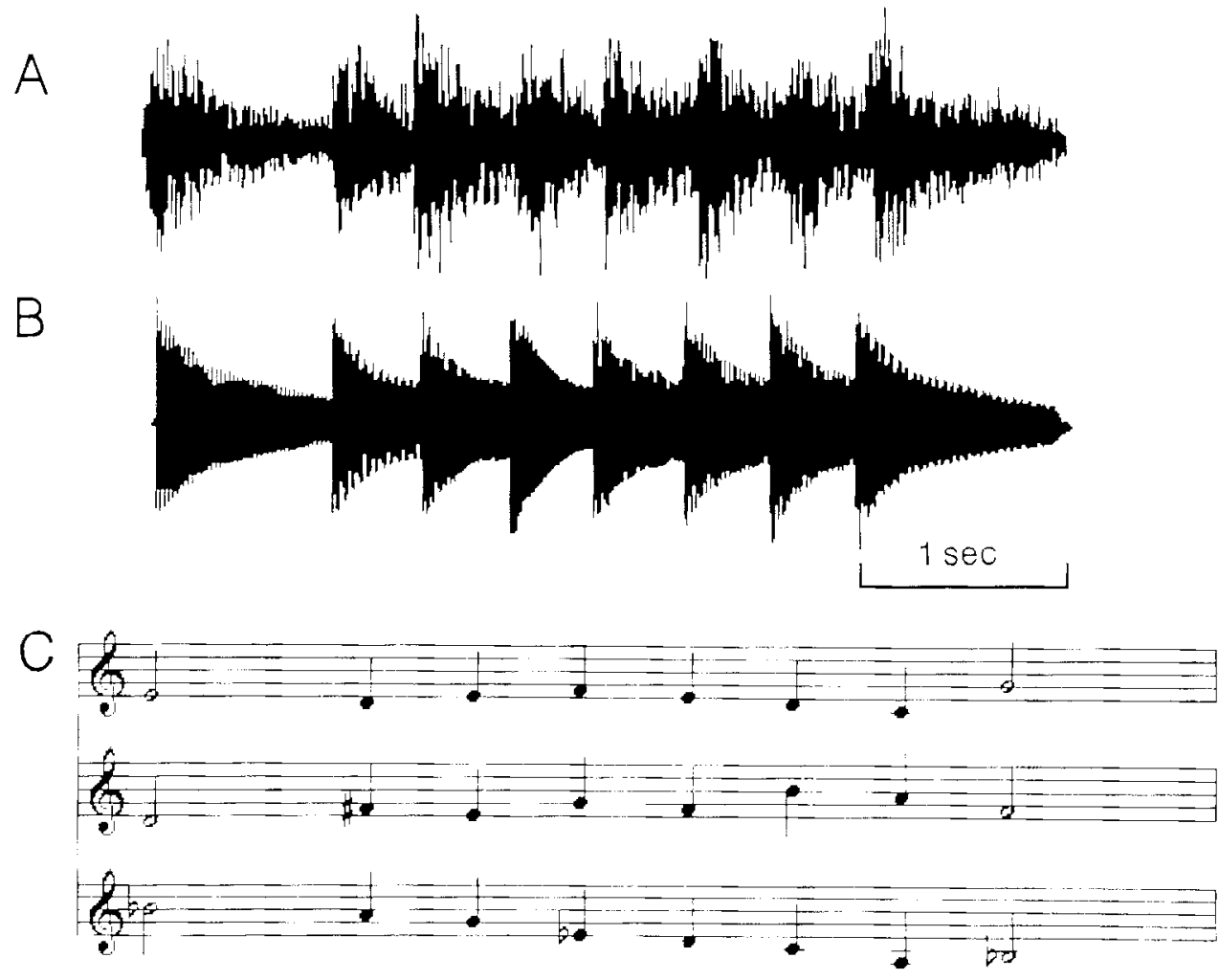

standardized stereotaxic courdirate system (Talairach and Tumnoux, 1988). PET images were reconstructed using a $20 \mathrm{~mm}$ Hanning filter to overcome residual anatomical variability, normalized for global CBF and averaged across subjects for each activation state. The mean statedependent change image volume was then obtained, and converted to a $t$ statistic volume by dividing each voxel by the mean standard deviation in normalized ( $\mathrm{BF}$ for all intracerebral voxels (Worsley et al., 1992). Individual MR images were subjected to the same averaging procedure, such that composite images volumes sampled at approximately $1.5 \mathrm{~mm}$ in each dimension were obtained for both $t$ statistic and MRI volumes. Anatomical and functional images were merged to allow direct localization on the MR images of regions with high $t$ values.

The presence of significant focal changes in CBF was tested by a method based on 3-D Gaussian random field theory (Worsley et al.. 1992). Values equal to or exceeding a criterion of $t=3.5$ were dcemed statistically significant ( $p<0.0002$, one-tailed, uncorrected). Correcting for multiplc comparisons, a $t$ value of 3.5 yields a false positive rate of only 0.58 in 200 resolution elements (each of which has dimensions 20 $\times 20 \times 7.6 \mathrm{~mm}$ ), which approximates the volume of cortex scanned.
Subjects. Twelve nomal right-handed volunteers, lialf of each sex. participated in the study after giving informed consent. Subjects were unselected for musical training; most of them had reccived some musical instruction, usually as part of their elementary or high school education. but none were professional musicians.

Stimuli. Two types of stimuli were prepared: noise bursts and melodies. The noise bursts were constructed so as to approximate the acoustic characteristics of the melodies in terms of number, duration, interstimulus presentation rate, intensity, and onset offset shape. White noise was synthesized on a MACII computer, and then segmented to match the average duration of each note of the melodies (see below), producing a "noise melody" consisting of eight noise segments with appropriate durations (see Fig. 1). The individual noise segments were further matched to the notes by shaping their onsets and offsets to approximate the amplitude envelopes of the musical tones, and the entire sequence was then attenuated to an intensity level similar to that of the melodies. Total duration of the noise pattern was $5.0 \mathrm{sec}$.

Sixtecn different 8-note tonal melodies were prepared, all identical in their rhythmic configuration (Fig. 1), with the aim of allowing pitch

Table 1. Summary of paradigm, showing stimuli presented and responses elicited during each of the four experimental conditions

\begin{tabular}{|c|c|c|c|c|}
\hline Condition & Stimulus & Response & $\begin{array}{l}\% \\
\text { correct }\end{array}$ & $\begin{array}{l}\text { Reac- } \\
\text { tion } \\
\text { time }\end{array}$ \\
\hline Noise & Noise bursts & Key press after each stimulus & - & - \\
\hline Passive melodies & 8-Note mclodies & Key press after each stimulus & - & - \\
\hline 2 Note & 8 Note melodies & Comparison of first two notes & 95.8 & 446 \\
\hline First/Last & 8-Note melodies & Comparison of first and last notes & 89.1 & 917 \\
\hline
\end{tabular}

The stimuli were identical for all but the first (noise) condition, and the notor response (key press) was the salue in all four conditions. The subjects' judgments differed across conditions. No specific decision was required for either of the first two conditions. However, in the 2-note condition the pitch of the first two notes was to be compared, and the appropriate decision signalled by a key press, whereas in the first/ast condition the pitch of the first note of the melody was to be compared to the pitch of the last note, followed by the appropriate key press. Performance data (mean percentage correct and mean reaction time, in milliseconds, measured from stimulus offset) were collected on line during PET scanning, and indicate that subjects successfully performed the task, and that the pitch comparison in the first/last condition was more difficult than in the 2 -note condition. 
Table 2. Passive melodies minus noise

\begin{tabular}{|c|c|c|c|c|c|}
\hline \multirow[b]{2}{*}{ Region } & \multirow{2}{*}{$\begin{array}{l}\text { Brodmann } \\
\text { area }\end{array}$} & \multicolumn{3}{|c|}{ Coordinates (mm) } & \multirow[b]{2}{*}{$t$ Value } \\
\hline & & $\mathrm{x}$ & $\mathrm{y}$ & $\mathrm{z}$ & \\
\hline \multicolumn{6}{|l|}{ Blood flow increases } \\
\hline 1. R Superior temporal gyrus & 22 & 62 & -25 & 3 & 4.47 \\
\hline 2. R Fusiform gyrus & 19 & 25 & -78 & -11 & 4.26 \\
\hline \multicolumn{6}{|l|}{ Blood flow decreases } \\
\hline 3. M Posterior cingulate gyrus & 31 & 4 & -49 & 29 & 4.40 \\
\hline 4. M Posterior cingulate gyrus & $31 / 7$ & 0 & -52 & 40 & 3.74 \\
\hline 5. R Frontal operculum & 44 & 44 & 10 & 12 & 3.64 \\
\hline 6. $\mathrm{R}$ Inferior colliculus & - & 7 & -26 & -17 & 3.53 \\
\hline
\end{tabular}

Data show significant activation foci (blood flow increases and decreases, in order of decreasing significance) for subtraction of passive melody condition minus noise condition. In this and subsequent tables, stereotaxic coordinates are derived from the human brain atlas of Talairach and Tournoux (1988), and refer, in millimeters, to medial-lateral position $(x)$ relative to midline (positive $=$ right), anterior-posterior position $(y)$ relative to the anterior commissure (positive $=$ anterior), and superior-inferior position $(\mathrm{z})$ relative to the commissural line (positive $=$ superior). Designation of Brodmann areas for cortical areas, based on this atlas, is approximate only. Significance level is given in $t$ test units (see Materials and Methods for details). L, left; $\mathrm{R}$, right; $\mathrm{M}$, midline.

judgments of either the first two notes, or the first and last notes. The duration of the first and last notes of each melody (approximately $1 \mathrm{sec}$ ) was twice that of each of the six intermediate notes, in order to facilitate their comparison. The last note had a higher pitch than the first in half of the 16 melodies, and in the other half, the last note was higher in pitch. Within each of these subsets of eight melodies, half contained a rising interval between the first and second notes, and the other half contained a falling interval (see Fig. 1). Thus, the pitch change between the first two notes was independent of the pitch change between the first and last notes. Furthermore, the average pitch distance of the notes to be compared in each condition was comparable across melodies: the first two notes differed in pitch by an average distance of 4.5 semitones (range, 1-12); the average pitch distance for the first and last notes was 5.5 semitones (range, 3-12). The melodies were played on a Yamaha PS4 electronic keyboard, using the "guitar" timbre, then digitized and stored on the computer for later playback. The average total duration of each melody was $4.7 \mathrm{sec}$.

Procedure. Four separate conditions were run during each of the four scanning periods (see Table 1). Although each scan lasted only $60 \mathrm{sec}$, the tasks were always begun several seconds before scanning commenced, and continued after scanning, until all 16 melodies had been presented. Performance data were collected on each subject on line during scanning. The total duration of each stimulus condition was usually about $2.5 \mathrm{~min}$.

During the first condition, termed the "noise" condition, subjects listened to the series of noise bursts described above, and after each "noise melody" depressed a key with their right hand, which resulted in the next stimulus sequence being played. In the second condition, termed "passive melodies," the subjects were presented with each of the 16 tonal melodies, and depressed a key after each one, as before. No overt judgments were required, but subjects were instructed to listen carefully to each melody as it was played. In the third condition, the "2-note" pitch comparison, subjects listened to the same melodies as before, but this time were instructed to determine whether the pitch of the second note was higher or lower than that of the first note. They were to respond accordingly on the computer keyboard, but were requested to withold the response until after the entire melody had been played. Finally, in the "first/last" pitch judgment, subjects were asked to compare the pitch of the first and last notes, ignoring the notes in between, and to respond as beforc according to whether the pitch rose or fell.

The order of the "2-note" and "first/last" tasks was counterbalanced across subjects, who were not instructed as to the nature of any of the judgments until immediately prior to scanning; however, several practice trials were given prior to starting each task. A different random order of the same 16 melodies was presented to each subject during each of the melody conditions. All stimuli were presented binaurally via insert earphones (Eartone type 3A). Subjects kept their eyes shut throughout the scanning period.

\section{Results}

The experiment was set up to permit specific comparisons, accomplished via subtraction of relevant conditions. The results of these subtractions, in terms of significant regions of CBF change (increases or decreases), are given in Tables $2-4$, together with stereotaxic coordinates based on the brain atlas of Talairach and Tournoux (1988).

The first comparison, passive melodies minus noise, permits examination of the cerebral regions specifically active during listening to novel tonal melodies, as opposed to the activation that might be present with any auditory stimulus with similar acoustic characteristics. The principal result, shown in Figure $2 A$, indicates a large CBF increase in the right superior temporal gyrus, anterior to the primary auditory cortex, as predicted. In addition, and unexpectedly, a significant focus was also identified in the fusiform gyrus of the right hemisphere, within area 19 (see Fig. $2 A$ ). An area of positive CBF change can also be seen in the left temporal lobe (Fig. $2 A ; \mathrm{x}, \mathrm{y}, \mathrm{z}$ coordinates: -52 , $-13,2)$, although the strength of this signal $(t=3.35)$ was insufficient to achieve statistical significance by our criteria. Areas of blood flow decrease were also observed in this subtraction, including the posterior cingulate region and right frontal operculum.

The second and third comparisons both use the passive melody condition as the baseline, so that any activation seen represents neural responses beyond those already present during initial listening to the same stimulus materials. Subtraction of the 2-note condition from passive melodies results in the pattern of cerebral activation shown in Figure $2 B$ and Table 3 . Most prominent in this subtraction is the significant activation within the right frontal lobe, as predicted. Two separate foci can be distinguished within distinct cytoarchitectonic regions, including Brodmann's areas 47/11 (focus 1), and 6 (focus 2). Other regions that were also active include anterior cingulate and cerebellum. An area within the medial parietal lobe, $8 \mathrm{~mm}$ to the right of midline, fell at the borderline of significance $(t=3.47)$.

Most notable among the areas showing significantly decreased $\mathrm{CBF}$ are two adjacent foci within the left gyrus of Heschl (foci 6 and 8 of Table 3), or primary auditory cortex, shown in Figure 

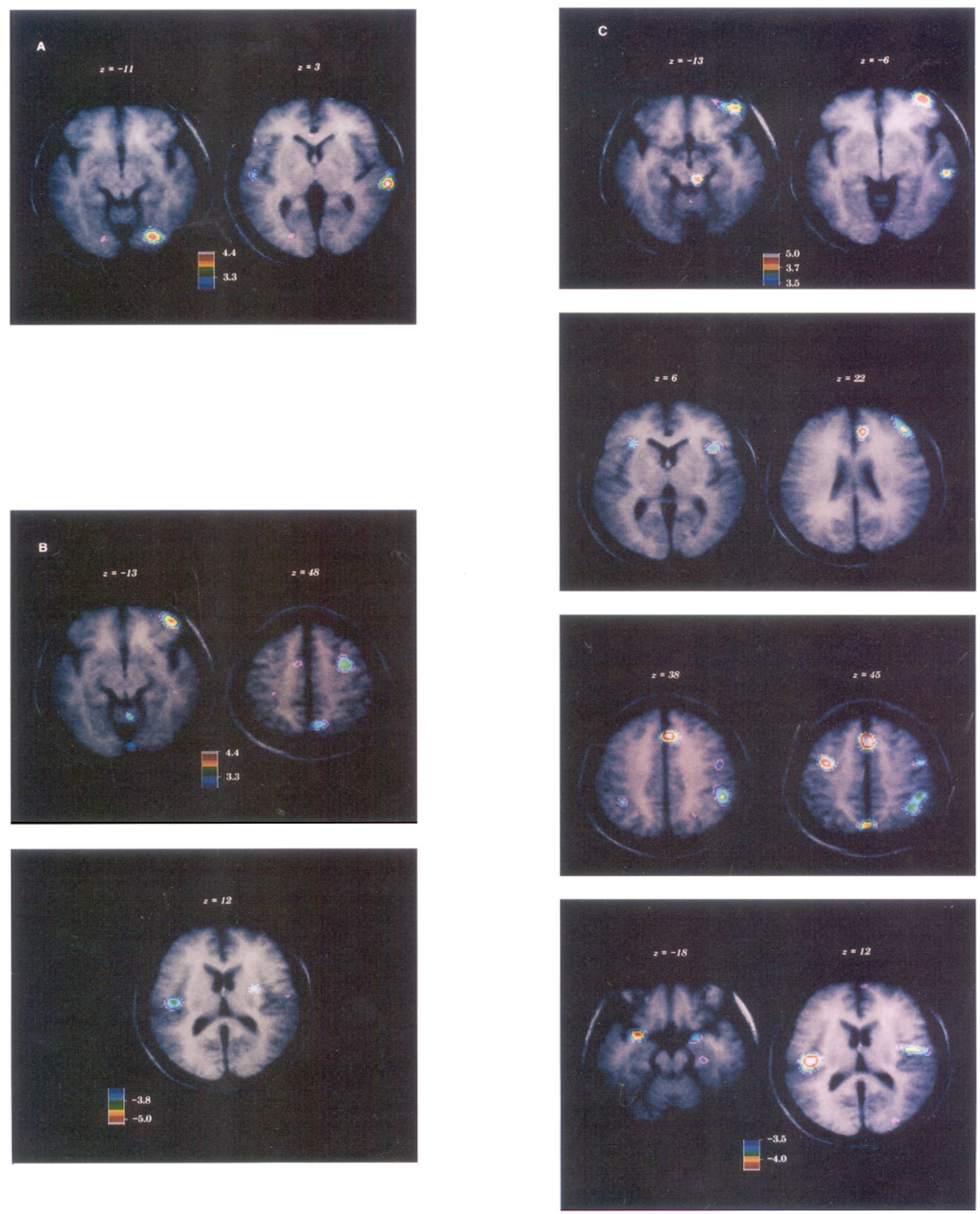

Figure 2. Selected cortical regions activated in the various conditions. The averaged PET subtraction images for the most pertinent foci are shown superimposed upon the corresponding averaged horizontal MRI scan. Subtraction of the control from activated state in each case yielded the focal changes in blood flow shown as a $t$ statistic image, whose range is coded by the color scale shown on each figure (see Table 1). A, passive melody minus noise subtraction. The two images in this figure, taken at horizontal levels of $11 \mathrm{~mm}$ below and $3 \mathrm{~mm}$ above the commissural plane, illustrate 
Table 3. 2-Note judgment minus passive melodies

\begin{tabular}{|c|c|c|c|c|c|}
\hline \multirow[b]{2}{*}{ Region } & \multirow{2}{*}{$\begin{array}{l}\text { Brodmann } \\
\text { area }\end{array}$} & \multicolumn{3}{|c|}{ Coordinates $(\mathrm{mm})$} & \multirow[b]{2}{*}{$t$ Value } \\
\hline & & $\mathrm{x}$ & $\mathrm{y}$ & 7 & \\
\hline \multicolumn{6}{|l|}{ Blood flow increases } \\
\hline 1. R Inferior frontal & $47 / 11$ & 40 & 46 & -13 & 4.15 \\
\hline 2. R Superior frontal & 6 & 31 & 1 & 48 & 3.81 \\
\hline 3. M Cerebellum & - & 4 & -57 & -11 & 3.81 \\
\hline 4. L Anterior cingulate gyrus & 32 & -8 & 18 & 29 & 3.66 \\
\hline \multicolumn{6}{|l|}{ Blood flow decreases } \\
\hline 5. M Anterior cingulate gyrus & 24 & -1 & 27 & -2 & 4.88 \\
\hline 6. L Heschl's gyrus & 41 & -43 & -18 & 12 & 3.89 \\
\hline 7. R Claustrum/putamen & - & 27 & -2 & 11 & 3.86 \\
\hline 8. L Heschl's gyrus & 41 & -44 & -14 & 11 & 3.86 \\
\hline 9. L Frontal pole & 10 & -5 & 58 & 0 & 3.82 \\
\hline 10. L Frontal pole & 9 & -15 & 60 & 30 & 3.67 \\
\hline 11. L Latcral cerebellum & - & -35 & -45 & -20 & 3.67 \\
\hline 12. L Lateral cerebellum & - & -20 & -47 & -12 & 3.63 \\
\hline
\end{tabular}

Data show significant activation foci (blood flow increases and decreases) for subtraction of 2-note condition minus passive melody condition. See Table 2 note for further details.

$2 B$. In addition, we observed decreases in the anterior cingulate cortex, in two left frontal polar regions, and in the left lateral cercbellum.

The first/last passive melodies subtraction also yielded several $\mathrm{CBF}$ increases within the right frontal lobe, consistent with the predictions (see Fig. $2 C$, focus 3 visible at $z=-13$ and $z=-6$, and focus 11 , at $z=22$ ). Also in keeping with our predictions, we identified an area of significant CBF increase within area 21 of the right temporal lobe (focus $6,7=6$ ), indicating that this condition resulted in greater activity within the right auditory association cortex than already present during passive listening to melodies.

The analysis of the data from this subtraction also yielded numerous other regions of positive activation, notably within the parietal lobe bilaterally, and on the right side (foci 12, 13 , and 14 , respectively, all visible in Fig. $2 C$ al $z=38$ and $z=$ 45 ), and medially (focus 7 , visible at $z=45$ in Fig. $2 C$ ). Other regions visible in Figure $2 \mathrm{C}$ are in the anterior cingulate gyrus $(\mathrm{z}=22, \mathrm{z}=38, \mathrm{z}=45)$, left precentral region $(\mathrm{z}=45)$, and right inferior colliculus $(z=-13)$. Bilateral foci were also observed deep within the Sylvian fissure, at the junction between the frontal opercular region and the insula (visible in Fig. $2 \mathrm{C}$ at $z=6)$

As in the previous subtraction, the first/last minus passive melody analysis also yielded a prominent area of CBF decrease within Heschl's gyrus in the left hemisphere (focus 19 in Table 4 , visible in the bottom row of Fig. $2 C$ at $z=12$ ). In addition, several other left-hemisphere regions demonstrated lower CBF, including an inferior temporal region (focus 21), and an area in the posterior parietal lobe (focus 24). Finally, it is interesting to note the presence of several foci of $\mathrm{CBF}$ decrease within the medial temporal region that may be related, including the uncus bilaterally (foci 23 and 25), and one in the right parahippocampal gyrus (focus 28), all of which are visible in the bottom row of Figure $2 C$ at $z=-18$.

The results of the subjects' performance on the 2-note and first/last conditions are given in Table 1, which shows the mean percentage correct, together with the mean reaction time. All subjects performed both tasks well above chance $(50 \%)$, but, as expected, more errors were committed on the first/last condition than the 2-note condition (Wilcoxon signed rank test, $W=45$, $p<0.01$ ), and reaction times were consistently longer.

\section{Discussion}

In general, the patterns of CBF changes observed in this study provide support for the hypothesis that perceptual analysis and short-term retention of tonal pitch information preferentially involve neural systems within the right frontal and temporal cortices. However, it also appears that the operations involved in these complex tasks make demands upon a widely distributed

the significant CBF increases noted in the fusiform gyrus (focus 2 in Table 2 ) and right superior temporal gyrus (focus 1), respectively. Note also the presence of a nonsignificant $C B F$ increase in the left superior temporal area, visible at $z=3 \mathrm{~mm}$. $B$. Two-note minus passive melody subtraction. Shown are the two significant areas of CBF increase in the right frontal cortex, one more inferior (focus 1 in Table 3; plane of image $z=-13$ ), and one more superior (focus 2; plane of image $z=48$ ), as well as a significant CBF decrease (second row) in the left primary auditory cortex (foci 6 and 8; plane of image $z-12$ ). C. First/last minus passive melody subtraction. This figure illustrates the following significant CBF increases. Top row, Increases in the right inferior frontal lobe (focus 3 in Table 4, visible at $z=-13$ and $z=-6$ ), in the right inferior colliculus (focus 5 , $z$ $=-13$ ), and in the right temporal lobe (focus $6, z=-6$ ). Second row, Increases bilaterally at the junction between anterior insular and frontal opercular regions (foci 15 and 16 , boll visible at $z=6$ ), in the right mid-frontal region (focus $11, z=22$ ), and in the right anterior cingulate gyrus (focus 2 , visible at $z=22$ ). Third row, Increases in the right and left inferior parietal lobe (foci 12 and $13, z=38$ ), in the right superior parietal region (focus 14, merging with the lower parietal-lobe focus at $z=45$ ), in the anterior cingulate. midline (focus 1 , visible at $z=38$ and $z=45$ ), in the left precentral region (focus $4, z=45$ ), and in the medial parietal cortex, midline (focus 7 , visible at $z=45$ ). Bottom row, Some of the significant $\mathrm{CBF}$ decreases detected in this subtraction: in the left and right uncus, and right parahippocampal gyrus (foci 23,25 , and 28 , all visible at $z=-18$ ), and in the left primary auditory cortex (focus $19, z=12$ ); also visible at $z=12$ is a portion of the decrease in the right opercular region (focus 22). 
Table 4. First/last note judgment minus passive melodies

\begin{tabular}{|c|c|c|c|c|c|}
\hline \multirow[b]{2}{*}{ Region } & \multirow{2}{*}{$\begin{array}{l}\text { Brodmann } \\
\text { area }\end{array}$} & \multicolumn{3}{|c|}{ Coordinates $(\mathrm{mm})$} & \multirow[b]{2}{*}{$t$ Value } \\
\hline & & $\mathrm{x}$ & $\mathrm{y}$ & $z$ & \\
\hline \multicolumn{6}{|l|}{ Blood flow increases } \\
\hline 1. M Anterior cingulate gyrus & $32 / 8$ & -1 & 24 & 42 & 4.94 \\
\hline 2. R Anterior cingulate gyrus & 32 & 5 & 36 & 26 & 4.94 \\
\hline 3. R Inferior frontal & 47 & 38 & 51 & -9 & 4.77 \\
\hline 4. L Precentral frontal & 6 & -35 & -2 & 45 & 4.41 \\
\hline 5. R Inferior colliculus & - & 7 & -30 & -13 & 4.29 \\
\hline 6. $\mathrm{R}$ Middle temporal gyrus & 21 & 55 & -26 & -6 & 4.04 \\
\hline 7. M Medial parietal & 7 & -1 & -69 & 47 & 4.04 \\
\hline 8. R Mid-frontal & 9 & 36 & 36 & 31 & 3.80 \\
\hline 9. R Cerebellum & - & 8 & -69 & -24 & 3.80 \\
\hline 10. L Mid-fiontal & $9 / 46$ & -36 & 29 & 30 & 3.76 \\
\hline 11. R Mid-frontal & $9 / 46$ & 39 & 41 & 22 & 3.72 \\
\hline 12. R Inferior parictal & 40 & 47 & -38 & 38 & 3.68 \\
\hline 13. L Inferior parietal & 40 & -36 & -47 & 39 & 3.68 \\
\hline 14. R Superior parietal & $40 / 7$ & 39 & -50 & 45 & 3.63 \\
\hline 15. R Insula/frontal operculum & 45 & 38 & 20 & 5 & 3.63 \\
\hline 16. L Insula/frontal operculum & 45 & -31 & 22 & 8 & 3.59 \\
\hline 17. M Cerebellum & - & 1 & -52 & -11 & 3.51 \\
\hline \multicolumn{6}{|l|}{ Blood flow decreases } \\
\hline 18. $M$ Anterior cingulate gyrus & 24 & -3 & 30 & -2 & 5.21 \\
\hline 19. L Heschl's gyrus & 41 & -42 & -19 & 12 & 4.76 \\
\hline 20. L Posterior cingulate gyrus & 31 & -7 & -47 & 35 & 4.60 \\
\hline 21. L Inferior temporal & 20 & -46 & -11 & -29 & 4.48 \\
\hline 22. R Parietal operculum & 40 & 46 & $-\cdots 18$ & 21 & 4.44 \\
\hline 23. L Uncus & $34 / 28$ & -29 & 5 & -18 & 4.23 \\
\hline 24. L Posterior parietal & 39 & -48 & -69 & 24 & 4.19 \\
\hline 25. R Uncus & $34 / 28$ & 23 & 5 & -17 & 3.86 \\
\hline 26. R Medial occipitotemporal & 37 & 27 & -56 & -6 & 3.82 \\
\hline 27. M Frontal pole & 10 & 3 & 61 & 15 & 3.58 \\
\hline 28. R Parahippocampal gyrus & $28 / 36$ & 28 & -25 & -20 & 3.50 \\
\hline
\end{tabular}

Data show significant activation foci (blood flow increases and decreases) for subtraction of first/last note condition minus passive melody condition. See Table 2 note for further details.

system, involving interactions between a number of distinct regions in both cerebral hemispheres. Some of the analyses yielded a large number of $C B F$ change foci, not all of which can be readily interpreted, given our present knowledge. In this section, therefore, we discuss the most pertinent results from each subtraction, and then turn to a more general discussion.

\section{Passive melodies minus noise}

As predicted, this comparison yielded activation of the right superior temporal cortex (focus 1 in Table 2, visible in Fig. $2 A$ at $z=3$ ), in keeping with the recognized role of this region in melodic processing. The most surprising finding in this analysis was the CBF increase in the right occipital cortex (fusiform gyrus) while subjects listened to melodies, as contrasted to listening to acoustically matched noise segments (visible in Fig. $2 A$ at $\mathrm{z}=-11$ ). The likelihood that this result represents simply a statistical artifact would appear low, since the $t$ value of 4.26 is well above threshold; it is also worth mentioning that a second focus within area 19 was observed, and although it did not reach significance $(t=3.25)$, its presence further supports the finding. The possibility that the effect is due to some extraneous visual stimulation is also excluded, since scanning was carried out with the subjects' eyes closed.
Area 19 is typically described as extrastriate visual cortex (Diamond et al., 1985); there is but scant physiological evidence for its direct participation in auditory processing (Morrell, 1973). Previous PET studies using melodies or musical scales have failed to observe occipital changes (Mazziota et al., 1982; Sergent et al., 1992); but the former study measured glucose metabolism with no specific task or stimulus controls, rendering interpretation difficult, while in the latter study the baseline condition included visual stimulation, rather than an acoustically appropriate control, so any visual-cortical activation might have been obscured. However, there does exist one brief report of a PET study using frequency-modulated tones in which activation of left area 19 was reported (Griffiths and Brown, 1991). These authors raise the possibility that a cross-modal spatial perceptual system was being engaged by the apparent movement of the frequency-modulated tones. Melodies, of course, involve frequency modulation; it is therefore plausible to suggest that at least some subjects may have activated visual representations, consciously or not, and that our observations in area 19 reflect this process. The notion that activation of this region in the present study is due to visual imagery processes would be consistent with recent findings of blood flow increases in area 19 and other visual cortical regions during explicit visual image 
generation tasks (Kosslyn et al., 1993). This possibility must remain a conjecture for the moment, until direct evidence can be adduced in its favor. We also do not know at present whether the putative visual activation is a general effect, or if it is specific to some particular global or local features of our stimulus materials.

The fact that the occipital CBF activity was confined to the right side is consistent with the general tendency for right-hemisphere processes to be particularly important in melody perception. This right-side predominance was also evident in the temporal-lobe activation, which, as predicted, was observed in the superior temporal cortex anterior and inferior to Heschl's gyri. The much weaker activation in the left temporal cortex (visible in Fig. $2 A$ at $\mathrm{z}=3$ ) is probably genuine, since both temporal lobes undoubtedly contain neurons responsive to the acoustic features present in the melodies. The asymmetry we observe likely reflects the specialization of neuronal networks within the right associative auditory cortices for perceptual analysis of tonal information, consistent with the human lesion evidence reviewed above (Milner, 1962; Zatorre, 1985; Zatorre and Halpern, 1993).

Note that, in this subtraction, no CBF change was present in the primary auditory cortices beyond that elicited in the control condition. This result is explained by the use of noise sequences as the control condition. Since (BF can change markedly with changes to physical stimulus parameters (Fox and Raichle, 1984), it can be problematic to compare scanning conditions in which the stimuli are not physically similar. In the present experiment, by using the acoustically matched "noise melodies" (see Fig. 1), nonspecific auditory processing could be dissociated from that which is uniquely elicited by listening to melodies. We previously demonstrated that similar noise bursts result in primary auditory cortical stimulation when contrasted to a silent condition (Zatorre el al, 1992). These findings, logether with findings from prior PET studies using speech sounds or tones (Lauter et al., 1985; Petersen et al., 1988; Wise et al., 1991; Demonet et al., 1992) point to differential activation of primary versus secondary auditory areas within the superior temporal gyrus, according to the nature of the processing elicited by a given stimulus. Although the noise stimuli proved successful in demonstrating the intended dissociation, caution must be still exercised in interpreting the results, for the noise bursts are clearly not physically identical to the melodic sounds. For example, the noise stimuli contain no periodicity, whereas the tones do; their spectral composition also is quite different. It remains to be established, therefore, which specific features of the melodies may lead to the observed pattern of activation.

A word is in order at this point about the interpretation to be given the "passive melodies" condition. Our use of the term "passive" is meant only to describe the lack of overt behavioral response, and does not imply that listening to such complex material is a passive cognitive process. On the contrary, it is certain that listening to melodies implies quite active, complex mental operations, including perceptual and mnemonic processing. (The subjects were, after all, instructed to listen carefully to the stimuli.) Apart from this purely semantic debate, however, questions can be raised about the validity of any condition in which the subject is not constrained to respond in a particular fashion. It might be argued, for example, that the lack of explicit task demands renders interpretation difficult, because it will not be possible to determine what specific operations were performed during the so-called passive task. It is clear that using specific behavioral tasks to identify particular aspects of processing is critically important, as will be discussed in the following section, but in many instances the question of interest is preciscly to understand the cognitive processes inherent to nondirected perceptual analysis. The advantage of functional PET brain mapping is that answers to this question can be inferred from the pattern of CBF changes during such "passive" perceptual conditions, as long as appropriate control conditions are used. Thus, in addition to providing relative ecological validity, a passive condition allows one to address this crucial question. In the present case, for example, we were interested in the cerebral mechanisms that allow extraction of perceptually relevant information from a tonal pattern, something that occurs automatically (Dowling and Harwood, 1986). If only experimenter-imposed judgments were to be analyzed, one might run the risk of overlooking or confounding the cerebral correlates of such automatic processes, which are likely to be of major importance in understanding music cognition in general.

\section{2-Note minus passive melodies}

We now turn to the results involving explicit pitch comparisons, which were carried out in two ways (2-note and first/last conditions). Note that in both cases the stimuli are identical, and that the judgment required (pitch rise or fall) is also the same, the only difference being which notes are to be compared. Thus, these conditions should allow us to study the cognitive processes required for pitch decisions under conditions of low or high memory load, respectively, using experimentally closely matched conditions. The performance data (Table 1) allow us to be certain that the subjects followed the instructions, and were indeed performing the appropriate comparisons, since performance rates were generally high. At the same time, we were able to document the expected decrease in correct performance comparing the 2-note to the first/last condition (Deutsch, 1970), which demonstrates that the latter comparison is indeed a more demanding cognitive task.

Considering first the 2-note minus passive melodies comparison, we had predicted that regions within the right frontal lobe would be activated, and this was indeed the case. Although the more superior frontal area (focus 2 in Table 3, visible in Fig. $2 B$ at $\mathrm{z}=48)$ is not far $(<2 \mathrm{~cm})$ from one reported by Zatorre et al. (1992), the more inferior one (focus $1, z=-13$ in Fig. $2 B$ ) is in a different cytoarchitectonic region (Brodmann arca $47 / 11)$.

In this same subtraction we also observed two adjacent areas of significant CBF decrease within the left primary auditory cortex, with no equivalent change in the homologous region on the right side (Fig. $2 B, \mathrm{z}=12$ ). Decreases in CBF may be simply interpreted as reflecting increases in the baseline condition over the experimental condition; that is, in this case the finding indicates that the left primary auditory cortex was significantly more active during the passive listening than during performance of the pitch judgment task. We shall return to this point below.

\section{First/last note minus passive melodies}

In the first/last minus passive melody comparison, we observed a greater number of separate foci of CBF change over a wider swath of cortical and subcortical territory than was evident in the low memory-load comparison, perhaps reflecting the complexity and increased cognitive demands of the task, as manifested in the increased error rate and slower reaction time. 
In particular, we note that our specific predictions were upheld by the presence of three lateral frontal peaks in the right hemisphere (Fig. 2C), only one of which (focus 8 ) is matched by a symmetric region in the left hemisphere (focus 10). In contrast to the 2-note condition, and also in accord with our predictions, we observed significantly increased CBF in the right temporal lobe (focus 6 , shown at $z=-6$ in Fig. $2 C$ ). This finding implies that additional processing was taking place within the right temporal region during performance of the pitch retention task, above and beyond that already accounted for in the passive melodies condition.

In the context of this task, it is reasonable to expect activation of many different cortical regions, as the first/last comparison involves a number of cognitive processes, in addition to shortterm retention. Thus, one may speculate that the numerous frontal-lobe sites observed might be associated with successful performance of distinct aspects of the task. For example, maintenance of pitch information in working memory might depend on a mechanism separate from that involved with the more "executive" functions required to monitor the presentation of the tones and their temporal order, and to direct the appropriate pitch comparison (see Milner and Petrides, 1984). Similarly, aspects of sustained attention that may rely on frontal-lobe mechanisms (Pardo et al., 1991) may also have been implicated in the present task. The right inferior colliculus, known to be an important auditory processing structure (Aitkin, 1986), was also activated in this subtraction (Fig. $2 \mathrm{C}, \mathrm{z}=-13$ ), indicating that it too is a component of a specialized distributed network involved in pitch memory.

This subtraction also yielded a significant CBF decrease in the left primary auditory region (bottom row of Fig. $2 C$ ), nearly identical to that found in the 2 -note minus passive melody subtraction. This unexpected finding strongly suggests a complex interaction in the physiological processes underlying performance of the tasks. As discussed in greater detail below, we propose that this finding reflects differential use of perceptual information computed at early stages of processing, within the primary auditory cortices. Since the right primary region is probably specialized for pitch extraction (Zatorre, 1988), the relative CBF decrease in the left primary region may indicate that information derived from this region is not required for either of the pitch-judgment tasks, whereas pitch information specifically derived from processing in the right primary auditory cortex is utilized.

The pattern of CBF change in the first/last condition as compared to passive listening also yielded significant CBF increases bilaterally near the junction of the frontal operculum and anterior insula (second row of Fig. $2 C$, at $\mathrm{z}=6$ ). We have recently obtained data on a vocal production task (Perry et al., 1993) in which we observed significant insular/opercular activation within 2-4 $\mathrm{mm}$ of those observed in the present investigation. These findings may therefore reflect the involvement of these regions in the control of vocal pitch, including possibly subvocal rehearsal. The latter possibility is consistent with the results of Paulesu et al. (1993), who report significant CBF increases in the insula bilaterally (albeit more posteriorly than in our studies) during covert rehearsal of visually presented letters.

Several areas of significant CBF increase were also found to lie within the parietal lobe: in the inferior parietal lobule bilaterally, and more superiorly on the right side (third row of Fig. $2 C$, at $\mathrm{z}=38$ and $\mathrm{z}=45$ ), as well as in medial parietal cortex, area 7 (Fig. $2 C, z=45$ ). These results clearly indicate a contri- bution from a parietal system to the performance of this task, but its precise nature can only be guessed at for the moment. It is tempting to speculate, given the widely acknowledged role of inferior parietal regions in spatial processing, that a recoding of pitch information may be taking place during the performance of this task. If our observation of CBГ changes in area 19 during passive listening does indeed reflect a visual component to the original percept, then perhaps the hypothesized recoding might involve integration of auditory and visual representations to permit some spatial comparison, which might facilitate the required pitch judgment.

Another possibility is that these parietal-lobe changes reflect some aspect of vigilance or sustained attention, which would undoubtedly accompany the first/last comparison. Pardo et al. (1991) have reported right parietal and dorsolateral frontal CBF increases in somatosensory and visual vigilance tasks; one of the parietal-lobe peaks in the present study (focus 12 in Table 2 ) is in close proximity (less than $6 \mathrm{~mm}$ ) to three of the points described by those investigators (one in each of their three tasks). Although mere similarity of location is not sufficient to infer functional similarity, these comparisons permit us to hypothesize that the task used in the present study may have cngaged part of the parietal component of the sustained attention network described by Pardo et al. (1991). In addition to the right parietal cortex's putative role in sustaining attention, its specific contribution to pitch processing mechanisms is partially supported by the recent study of Demonet et al. (1992), who found right inferior parietal and temporal activation using a tonal pitch task. Direct comparison of those data with our own is rendered difficult by the very different subtraction conditions used; the parietal sites identified in that study were also more inferiorly and anteriorly located than those found in the present study. Nonetheless, the fact that right-sided parietal and temporal regions were identified suggests that some of the same psychological processes may be implicated.

Finally, among the blood flow decreases in this subtraction we note a pattern that may be of importance: three regions within the medial temporal lobe were identified, in the uncus bilaterally, and in the parahippocampal gyrus on the right (see bottom row of Fig. $2 \mathrm{C}, \mathrm{z}=-18$ ). Very similar CBF decreases were also revealed by a directed search of the 2-note minus passive melody subtraction: bilaterally in the uncus (coordinates $19,3,-20$, and $-29,5,-15 ; t$ values, 3.25 and 3.17 , respectively), and in the right parahippocampal region (coordinates $29, \quad 19,18 ; t$ value, 2.98). Although these latter points did not reach our level of statistical significance, their close proximity to the significant areas in the other subtraction render them unlikely to be spurious. The medial temporal lobe has long been known to be associated with memory function in both humans (e.g., Milner, 1978) and monkeys (e.g., Mishkin, 1982). We have also obtained evidence from work with temporal lobectomy patients that regions within both temporal lobes are implicated in melodic memory processes, but that under some circumstances greater deficits are produced by lesions of the right temporal lobe than of the left (Samson and Zatorre, 1991b, 1992). We may therefore speculate that the CBF decreases observed reflect some aspect of mnemonic processing of these melodies. The CBF decrease indicates that greater CBF occurred during passive listening than during either pitch judgment task; it seems plausible that more automatic mnemonic encoding may occur during initial listening than when the subject's attention is drawn to a specific judgment. 


\section{General discussion and conclusion}

The tendency for right-asymmetric activation in frontal and temporal-lobe sites was observed in all three tasks; together with the relative decrease in the left primary region in the two-pitch judgment tasks, thesc findings strongly support our contention of a functional specialization within the right cerebral hemisphere for tonal melodic processing, in accord with considerable human neuropsychological evidence. The data permit us to distinguish between perceptual analysis mechanisms, involving primarily temporal neocortex, and auditory working memory mechanisms, involving complex temporofrontal interactions.

A preliminary uutline of a model to describe the perceptual processing stage may be suggested. Based on the results of the present study, together with the physiological and lesion literature discussed earlier, we may speculate that the primary auditory cortex is chiefly involved in early stages of processing (which might include computation of such signal parameters as pitch, duration, intensity, and spatial location), whereas more complex feature extraction, involving temporally distributed patterns of stimulation, is performed via populations of neurons within the secondary cortices. Neuronal systems located in both temporal lobes likely participate in higher-order perceptual analysis of melodies, but those on the right seem to be particularly important, perhaps because they are specialized to extract the features that are most relevant for melodic stimuli (including, e.g., invariant pitch-interval relationships, and spectral characteristics important for pitch and timbre perception). The existence of temporal-lobe neurons with complex response properties (e.g., McKenna et al., 1989) would be in keeping with this idea.

Note that such a hierarchical scheme need not imply a serial organization; indeed, both the anatomy and behavioral data reviewed earlier suggest that various stages of processing may occur in parallel. Furthermore, the presence of significant CBF decreases in the left primary auditory cortex implies that a simple linear additive model, in which a small number of operations are added by each subsequent task without affecting operations involved in earlier stages (Petersen et al., 1988), may be untenable. Instead, the CBF decreases observed in the two-pitch judgment conditions suggest that neural processes in the primary cortices may differ, depending upon the ultimate use of the extracted information. Thus, we may hypothesize that during active listening, in which pitch information, specifically, must be acted upon, there is an interaction between higher-order mechanisms (perhaps involving frontal-lobe structures) and lower-order systems (primary cortex), such that only the most relevant stimulus features are selected for further processing. It has previously been demonstrated that computation of complex-tone pitch depends crucially upon the right primary auditory cortex (Zatorre, 1988). We may therefore tentatively conclude that this feature of the stimulus is most important during the pitch judgment task, and that whatever information is derived from the left primary auditory region is less relevant, leading, therefore, to a relative decrease of CBF. Whether this explanation requires active suppression of information or not remains to be established. Note that no $\mathrm{CBF}$ change (positive or negative) was observed in the right primary auditory region during the two pitch judgment tasks. Therefore, it would seem that the perceptual information required was already extracted, probably automatically, during the passive listening stage.

In both pitch judgment conditions we observed significant
CBF increases within the right frontal cortex. Only in the high memory-load condition, however, did we observe an additional $\mathrm{CBF}$ increase in the right temporal lobe, beyond that seen in passive listening. We interpret this result, together with the right frontal activation, as evidence that the high memory load imposed by the first/last task engaged a specialized auditory working memory system, and that this system is instatiated in the brain via interaction of inferior frontal and superior temporal cortices in the right cerebral hemisphere. This conclusion would be in accord with an earlier study (Zatorre and Samson, 1991), in which deficits in pitch retention were observed after right frontal and/or temporal-lobe lesions. Further converging evidence favoring an asymmetric mechanism in pitch short-term memory comes from recent data collected using magnetenocephallography (MEG). Kaufman et al. (1991) report that MEG suppression time recorded over the right hemisphere correlated with stimulus set size for a short-term memory scanning task using tones. Moreover, they found an asymmetry in the amplitude variation of the $\mathrm{N} 100 \mathrm{~m}$ component of the magnetic evoked response, which originates in the auditory cortex, suggesting that early stages of cognitive processing, prior to memory search, are also linked to the right auditory region.

The present data are in accord with a detailed model for the interaction of frontal and temporal mechanisms proposed by Perry (1990; Marin and Perry, in press), who suggests that the connectivity of the superior temporal gyrus with the frontal cortex (Petrides and Pandya, 1988) may be one component of a distributed neural network that maintains auditory information in working memory. Thus, sensory information would be retained while some other process is carried out (in the present case the pitch of the first note is retained while the subject monitors for the occurrence of the final note). According to the model, this process would require neuronal interactions between the temporal neocortex, which has processed the pitch information, and portions of frontal cortex, which would actively maintain the appropriate information until the right time to make use of it. This conclusion is further supported by recent PET experiments (Petrides et al., 1993) implicating dorsolateral frontal-lobe mechanisms in monitoring of verbal information in working memory.

\section{References}

Aitkin LM (1986) The Auditory Midbrain. Clifton, NJ: Humana Press. Brugge JF, Reale RA (1985) Auditory cortex. In: Cerebral cortex, Vol 4 (Peters A, Jones EG, eds), pp 229-271. New York: Plenum.

Burton H, Jones EG (1976) The posterior thalamic region and its cortical projection in new world and old world monkeys. J Comp Neurol 168:249-302.

Celesia G (1976) Organization of auditory cortical areas in man. Brain 99:403-414.

Chavis D, Pandya DN (1976) Further observations on corticofrontal pathways in the rhesus monkey. Brain Res 117:369-386.

Colombo M, D'Amato MR, Rodman HR, Gross CG (1990) Auditory association cortex lesions impair auditory short-term memory in monkeys. Science 247:336-338.

Démonet J-F, Chollet F, Ramsay S, Cardebat D, Nespoulous J-L, Wise R, Rascol A, Frackowiak R (1992) The anatomy of phonological and semantic processing in normal subjects. Brain 115:1753-1768.

Deutsch D (1970) Tones and numbers: specificity of interference in short-term memory. Science 168:1604-1605.

Deutsch D (1982) The processing of pitch combinations. In: The psychology of music (Deutsch D, ed). New York: Academic.

Diamond IT, Fitzpatrick D, Sprague JM (1985) The extrastriate visual cortex. In: Cerebral cortex, Vol 4 (Peters $A$, Jones EG, eds), pp $63-$ 87. New York: Plenum. 
Divenyi PL, Robinson AJ (1989) Nonlinguistic auditory capabilities in aphasia. Brain Lang 37:290-326.

Dowling WJ, Harwood DL (1986) Music cognition. Orlando, FL: Academic.

Evans AC, Thompson CJ, Marrett S, Meyer E, Mazza M (199la) Performance evaluation of the PC-2048: a now 15-slice encodedcrystal PET scanner for neurological studies. IEEE Trans Med Imaging 10:90-98.

Evans AC, Marrett S, Torrescorzo J, Ku S, Collins L (199lb) MRI$\mathrm{PET}$ correlation in three dimensions using a volume-of-interest (VOI) atlas. J Cereb Blood Flow Metab 11:A69-A78.

Evans AC, Marrett S, Neelin P, Collins L, Worsley K, Dai W, Milot S, Meyer E, Bub D (1992) Anatomical mapping of functional activation in stereotactic coordinate space. Neuroimage 1:43-53

Evarts EV (1952) Effect of auditory cortex ablation on frequency discrimination in monkcy. J Neurophysiol 15:443-448.

Ferrier D (1876) The functions of the brain. London; Smith Elder.

Flechsig PE (1896) Gehirn und Seele. Leipzig: Veit.

Fox PT, Raichle ME (1984) Stimulus rate dependence of regional cerebral blood flow in human striate cortex, demonstrated by positron emission tomography. J Neurophysiol 51:1109-1120.

Galaburda A, Sanides F (1980) Cytoarchitectonic organization of the human auditory cortex. J Comp Neurol 190:597-610.

Gottlieb Y, Vaadia E, Abeles M (1989) Single unit activity in the auditory cortex of a monkey performing a short term memory task. Exp Brain Res 74:139-148.

Griffiths TD, Brown WD (1991) Focal activation of human left area 19 during an auditory task. J Cereb Blood Flow Metab 11 [Suppl 2]: S374.

Gross CG, Weiskrantz L (1962) Evidence for dissociation of impairment on auditory discrimination and delayed response following lateral frontal lesions in monkeys. Exp Neurol 5:453-476.

Heffner HE, Mastertion B (1978) Contribution of auditory cortex to hearing in the monkey (Macaca mulatta). In: Recent advances in primatology, Vol 1, Behaviour (Chivers DJ, Herbert J, eds). New York: Academic.

Iversen SD, Mishkin M (1973) Comparison of superior temporal and inferior prefrontal lesions on auditory and non-auditory tasks in rhesus monkeys. Brain Res 55:355-367.

Jerison HJ, Neff WD (1953) Effect of cortical ablation in the monkey on discrimination of auditory patterns. Fed Proc 12:237.

Kaufman L, Curtis S, Wang J-Z, Williamson SJ (1991) Changes in cortical activity when subjects scan memory for tones. Electroencephalogr Clin Neurophysiol 82:266-284.

Kosslyn SM, Alpert NM, Thompson WL, Maljkovic V, Weise SB, Chabris CF, Hamilton SE, Rauch SL, Buonanno FS (1993) Visual mental imagery activates topographically organized visual cortex: PEI investigations. J Cog Neurosci 5:263-287.

Lauter JL, Herscovitch P, Formby C, Raichle ME (1985) Tonotopic organization in human auditory cortex revealed by positron emission tomography. Hearing Res 20:199-205.

Iiegenis-Chauvel C, Musolino A. Chauvel P (1991) Localization of the primary auditory area in man. Brain 114:139-153.

Manley JA, Müller-Preuss P (1978) Response variability of auditory cortex cells in the squirrel monkey to constant acoustic stimuli. Exp Brain Res 32:171-180.

Marin OSM, Perry DW (in press) Neurological aspects of music perception and performance. In: The psychology of music, $2 \mathrm{~d}$ ed (Deutsch $D$, ed), in press. New York: Academic.

Mazziota JC, Phelps ME, Carson RE, Kuhl DE (1982) Tomographic mapping of human cerebral metabolism: auditory stimulation. Neurology 32:921-937.

McKenna TM, Weinberger NM, Diamond DM (1989) Responses of single auditory cortical neurons to tone sequences. Brain Res 481: 142-153.

Merzenich MM, Brugge JF (1973) Representation of the cochlear partition on the superior temporal plane of the macaque monkey. J Neurophysiol 24:193-202.

Milner B (1962) Laterality effects in audition. In: Interhemispheric relations and cerebral dominance (Mountcastle VB, ed), pp 177-195. Baltimore: Johns Hopkins.

Milner B (1978) Clues to the cerebral organization of memory. In: Cerebral correlates of conscious experience (Buser P, Rougeul-Buser A, eds), pp 139-153. Amsterdam: Elsevier.
Milner B, Petrides M (1984) Behavioural effects of frontal-lobe lesions in man. Trends Neurosci 7:403-407.

Mishkin M (1982) A memory system in the Monkey. Philos Trans R Soc Lond [Biol] 298:85-95.

Morrell F (1973) Visual system's view of acoustic space. Nature 238: 44-45.

Pardo JV, Fox PT, Raichle ME (1991) Localization of a human system for sustained attention by positron emission tomography. Nature 349 : 61-64.

Paulesu F, Frith CD, Frackowiak RSJ (1993) The neural correlates of the verbal component of working memory. Nature 362:342-345.

Penfield W, Jasper HH (1954) Epilepsy and the functional anatomy of the human brain. Boston: Little, Brown.

Penfield W, Perot P (1963) The brain's record of auditory and visual experience: a final summary and discussion. Brain 86:595-696.

Peretz I (1993) Auditory agnosia a functional andalysis. In: Thinking in sound: the cognitive psychology of human audition (McAdams S. Bigand E, eds), pp 199-230. London: Oxford UP.

Perry DW (1990) Ear and hemisphere difterences in melody recall. Dissert Abstr Int 52:552B (University microfilms, 91-17914).

Perry DW, Alivisatos B, Evans AC, Meyer E, Petrides M, Zatorre RJ (1993) Neural network supporting auditory-vocal integration in singing. J Acoust Soc Am 93:2403.

Petersen SF, Fox PT, Posner MI, Mintun M, Raichle ME (1988) Positron emission tomographic studies of the cortical anatomy of single word processing. Nature 331:585-589.

Petrides M. Pandya DN (1988) Association fiber pathways to the frontal cortex from the superior temporal region in the rhesus monkey. J Comp Neurol 273:52-66.

Petrides M, Alivisatos B, Meyer M, Evans AC (1993) Functional activation of the human frontal cortex during the performance of verbal working memory tasks. Proc Natl Acad Sci USA 90:878-882.

Raichle ME, Martin WRW, Herscovitch P, Mintun MA, Markham J (1983) Brain blood flow measured with intravenous H2015. II. Implementation and validation. J Nucl Med 24:790-798.

Robin DA, Tranel D, Damasio H (1990) Auditory perception of temporal and spectral events in patients with focal left and right cerebral lesions. Brain Lang 39:539-555.

Samson S, Zatorre RJ (1988) Discrimination of melodic and harmonic stimuli after unilateral cerebral excisions. Brain $\operatorname{Cogn} 7: 348-360$.

Samson S, Zatorre RJ (1991a) Timbre perception after unilateral tem. poral lobectomy in humans. Soc Neurosci Abstr 17:867.

Samson S, Zatorre RJ (1991b) Recognition memory for text and melody of songs after unilateral temporal-lobe lesion: evidence for dual encoding. J Exp Psychol [Learn Mcm Cogn] 17:793-804.

Samson S, Zatorre RJ (1992) Learning and retention of melodic and verbal information after unilateral temporal lobectomy. Neuropsychologia 30:815-826

Sergent J, Zuck E, Terriah S, MacDonald B (1992) Distributed neural network underlying musical sight-reading and keyboard performance. Science 257:106-109.

Shankweiler D (1966) Effects of temporal-lobe damage on the perception of dichotically presented melodies. J Com Physiol Psychol 62:115-119.

Sidtis JJ, Volpe BT (1988) Selective loss of complex-pitch or speech discrimination after unilateral lesion. Brain Lang 34:235-245.

Stepien LS, Cordeau JP, Rasmussen T (1960) The effect of temporal lobe and hippocampal lesions on auditory and visual recent memory in monkeys. Brain 83:470-489.

Talairach J, Tournoux P (1988) Co-planar stereotaxic atlas of the human brain. New York: Thieme.

Tramo MJ, Gazzaniga M (1989) Discrimination and recognition of complex harmonic spectra by the cerebral hemispheres: differential lateralization of acoustic-discriminative and semantic-associative functions in auditory pattern perception. Soc Neurosci Abstr 15:1060.

Tramo MJ, Bharucha JJ, Musiek FE (1990) Music perception and cognition following bilateral lesions of auditory cortex. J Cogn Neurosci $2: 195-212$.

Whitfield IC, Evans EF (1965) Responses of auditory cortical neurones to stimuli of changing frequency. J Neurophysiol 28:655-672.

Wise RJ, Chollet F, Hadar U, Friston K, Hoffner E, Frackowiak R (1991) Distribution of cortical neural networks involved in word comprehension and word retrieval. Brain 114:1803-1817.

Woolsey CN (1971) Tonotopic organization of the auditory cortex. 
In: Physiology of the auditory system (Sachs MB, ed), pp 271-282. Baltimore: National Educational Consultants.

Worslcy KJ, Evans AC, Marrett S, Neelin P (1992) A three-dimensional statistical analysis for CBF activation studies in human brain. J Cereb Blood Flow Metab 12:900-918.

Zatorre RJ (1985) Discrimination and recognition of tonal melodies after unilateral cerebral excisions. Neuropsychologia 23:31-41.

Zatorre RJ (1988) Pitch perception of complex tones and human temporal-lobe function. J Acoust Soc Am 84:566-572.
Zatorre RJ, Halpern AR (1993) Effect of unilateral temporal-lobe excision on perception and imagery of songs. Neuropsychologia 31: 221-232.

Zatorre RJ, Samson S (1991) Role of the right temporal neocortex in retention of pitch in auditory short-term memory. Brain 114:24032417.

Zatorre RJ, Evans AC, Meyer E, Gjedde A (1992) Lateralization of phonetic and pitch processing in speech perception. Science 256:846849. 